\title{
Multivariate FIGARCH and long memory process: evidence of oil price markets
}

\author{
Nadhem Selmi* and Nejib Hachicha
}

Department of Quantitative Methods, Faculty of Economics and Management of Sfax - Tunisia

\begin{tabular}{l}
\hline C H R O N I C L E \\
\hline Article history: \\
Received March 25, 2015 \\
Received in revised format June \\
12015 \\
Accepted June 172015 \\
Available online \\
June 22 2015 \\
\hline Keywords: \\
FIGARCH \\
ARFIMA \\
Oil price \\
MGARCH-DCC
\end{tabular}
\begin{abstract}
A B S T R A C T
Oil price markets can benefit from a better considerate of how shocks can affect volatility through time. This study assesses the impact of structural changes and outliers on volatility persistence of two crude oil markets WTI and Brent oil price between January 1, 1996 and March 17, 2014. First, we identify the FIGARCH process proposed by Baillie et al. (1996) [Baillie, R.T., Bollerslev, T., \& Mikkelsen, H.O., (1996), Fractionally integrated generalized autoregressive conditional heteroscedasticity. Journal of Econometrics, 74, 3-30.] and investigate some of its statistical proprieties and then incorporate this information into the volatility modelling. We also show that outliers can bias the estimation of the persistence of the volatility. Taking into account outliers on the volatility modelling process improve the understanding of volatility in crude oil markets.
\end{abstract}

\section{Introduction}

The objective of this paper is to provide econometrics and statistics properties of fractionally integrated GARCH (FIGARCH). The FIGARCH process implies a finite persistence of volatility shocks as there is no persistence in the GARCH model. Nonetheless, an IGARCH model implies a total persistence of shock. The price of Brent is one of the world's most important global economic signs. Policy-makers, producers, consumers and financial members observe its behavior. Since the end of the 1990s oil prices have been progressively growing reflecting rising demand for WTI and Brent oil price, particularly from developing countries. Crude oil has been very volatile, varying their courses and behavior either during the crisis or no-crisis circumstances. Studying the performance of volatility in crude oil prices is important for pricing oil price, for implementing hedging strategies and for assessing regulatory offers to restrict global capital flows. For example, varies in volatility can affect the risk exposure of industrial consumers and producers of Brent. These differences may alter their respective investments in oil price WTI and Brent oil price inventories and facilities for production and transportation. Crude oil prices are measured by high volatility in addition to a quantity of extreme shocks, such as the day Operation Desert Storm with a negative return of $-42 \%$ for WTI (Askari \& Krichene, 2008; Larsson \& Nossman, 2011). Financial and economic markets' members and policy-makers can profit from a better insight on how shocks can affect volatility over time, particularly whether the shocks of volatility are

\footnotetext{
* Corresponding author.

E-mail address: nadhem.selmi@yahoo.fr (N. Selmi) 
persistent. ARCH processes, developed by Engle (1982) and extended to GARCH by Bollerslev (1986) have been developed to detain two most important stylized facts of returns of WTI and Brent, which are heavy-tailed distribution and volatility clustering. According to these processes, the information accessible in a period is important to determine ting future variance. It is important to consider how the accessible information affects prevision uncertainty as the forecast possibility increases; in other words, the degree of persistence. Persistence in the variance evolving between instances refers to the property of momentum in conditional variance; i.e. past volatility explains present volatility.

Oil prices lighter than Brent, therefore easier to refine, the price per barrel of WTI was traditionally about 1 dollar higher than the Brent and \$ 2 above the OPEC basket but this generally tends to disappear. The price of WTI fell below that of Brent: once between May and November 2008, with a price difference of approximately 3 to $\$ 4$; a second time from the beginning of 2011, when the price gap was increasing gradually to 10 dollars apart in April 2011, then 20 dollars away in August 2011. September 5, 2011, the gap was 26.80 dollars (WTI to 83.60 dollars a barrel against \$ 110 for Brent). This growing gap can be explained by oil surpluses in the North American west. No infrastructure allows transport Canadian oil to eastern Canada (Ontario and Quebec) or south of the United States (Texas) which limits demand while supply is rising. The Keystone project and the Trans-Canada Pipeline would rebalance the price. Since the mid-1980s, WTI oil price has been principally influenced by the supply and demand condition in oil markets but not as strictly controlled. Although, the market made the WTI and Brent oil price frequently volatile with extensive amplitude. It can be found that there are significant extreme market risks that have led oil market investors to continue heavy possible losses. Consequently, there has been much interest in the oil market risk, and its measurement. To date, several literatures regarding oil market risk have discussed several topics from a qualitative perspective. For instance, what kinds of risk there are, what impact they have and what we should respond to them, are typical exploratory questions ${ }^{1}$. Evidently, it is important for us to know the characteristics of oil market risks. However, empirical studies are extremely scant.

The remainder of this paper is organized as follows. Section 2 describes the econometric methodology employed to prove the persistence of chocks face to the financial crisis. Section 3 describes the data and empirical results. The last section concludes.

\section{Econometric methodology}

\subsection{Process and main results}

In order to detain the long-memory component in the mean, Granger and Joyeux (1980) and Hosking (1981) independently formulated the Fractionally Integrated ARMA model (ARFIMA)

$$
\varphi(L)(1-L)^{d_{A R F M A}}\left(x_{t}-\alpha_{0}\right)=\theta(L) \varepsilon_{t}
$$

where $\varphi(L)=1-\sum_{j=1}^{k} \varphi_{j} L^{j}, \theta(L)=1+\sum_{j=1}^{z} \theta_{j} L^{j}, \alpha_{0}$ is the mean of $x_{t}$ and $\varepsilon_{t}$ is the white noise. In the same way Baillie et al. (1996) introduced the Fractionally Integrated GARCH (FIGARCH) model there by generalizing the GARCH and IGARCH qualifications. They described the conditional variance $h_{t}=\operatorname{Var}\left[\varepsilon_{t} \mid I_{t-1}\right]$ where $I_{t-1}$ is the information put in time $t-1$. The GARCH process might be written as: $[1-B(L)] h_{t}=w+\alpha(L) \varepsilon^{2}$,

where $\alpha(L)$ and $B(L)$ are lag polynomials of order $q$ and $p$, correspondingly and the skedastic innovation is defined as follows,

$$
v_{t}=\varepsilon_{t}^{2}-h_{t} \text {. }
$$

\footnotetext{
${ }^{1}$ See for example Li et al., 2005 and Huang et al., 2005.
} 
This can be accepted as an $\operatorname{ARMA}(m, p)$ process in $\varepsilon_{t}^{2}$

$[1-\beta(L)-\alpha(L)] \varepsilon_{t}^{2}=w+[1-\beta(L)] v_{t}$,

where $m=\max (q, p)$. The corresponding FIGARCH $(\mathrm{p}, \mathrm{d}, \mathrm{r})$ process is specified as follows,

$$
\phi(L)(1-L)^{d_{\text {FIGARCH }}} \varepsilon_{t}^{2}=w+[1-\beta(L)] v_{t},
$$

where

$\phi(L)=[1-\beta(L)-\alpha(L)](1-L)^{-d_{F I G A R C H}}$. Note that for $d_{\text {FIGARCH }}>0, \phi(L)$ is in principle of infinite order. The FIGARCH model reduces to a GARCH model when $d=0$ and to integrated (IGARCH) process when $d_{\text {FIGARCH }}=1$. The conditional variance of the FIGARCH model can be written as:

$h_{t}=\frac{w}{1-\beta(1)}+\lambda(L) \varepsilon_{t}^{2}$ for the univariate case where $\lambda(L)=1-\left\{\left[\phi(L)(1-L)^{d}\right] /[1-\beta(L)]\right\}$.

For the multivariate cases

$$
h_{11, t}=\alpha_{11} \varepsilon_{1, t-1}^{2}+\beta_{11} h_{11, t-1}+w_{1} \text {, and } h_{22, t}=\alpha_{11} \varepsilon_{2, t-1}^{2}+\beta_{22} h_{22, t-1}+w_{2} \text {, }
$$

and

$$
h_{12, t}=\rho\left(h_{11, t}, h_{22, t}\right)^{1 / 2} \text {. }
$$

This specification is very parsimonious. Positive definiteness is guaranteed provided $|\rho|<1$ and $\alpha_{j j}$, $\beta_{j j}$ and $w_{j}(\mathrm{j}=1,2)$ are such that $h_{11, t}$ and $h_{22, t}$ are always positive ${ }^{2}$.

Breidt et al. (1998), provided the GARCH (p,q) process written as $[1-\alpha(L)-\beta(L)]\left(\sigma_{t}^{2}-\sigma^{2}\right)=\alpha(L) v(t)$, with $\sigma^{2}$ is the unconditional variance of $\varepsilon_{t}$ a model GARCH. As so the FIGARCH $\left(\left(p, d_{\text {FIGARCH }}, q\right)\right.$ model can be written as

$$
\phi(L)(1-L)^{d_{\text {FIGARCH }}}\left(\sigma_{t}^{2}-\sigma^{2}\right)=\alpha(L) v_{t}
$$

The fractional difference operator is defined based on the Maclaurin series as:

$$
(1-L)^{d_{\text {ARFMA }}}=1-d_{\text {ARFIMA }} \sum_{j=1}^{\infty} \frac{\Gamma\left(j-d_{\text {ARFIMA }}\right)}{\Gamma(j+1) \Gamma\left(1-d_{\text {ARFIMA }}\right)} L^{j}=1-\sum_{\mathrm{j}=1}^{\infty} \pi_{\mathrm{j}}\left(d_{\text {ARFIMA }}\right) L^{j} .
$$

The constant in the FIGARCH model is deferential to that of the constant in the ARFIMA model. Parameter $d_{\text {ARFIMA }}$ varies over the interval $[-0.5,0.5]$ while $d_{\text {FIGARCH }}$ varies over the interval $[0 ; 1]$.

- if $0<d_{\text {ARFIMA }}<0.5$, the ARFIMA model is a stationary process with long memory. The autocorrelations are positive, decrease hyperbolically and tend to zero when the delay increases.

- if $d_{\text {ARFIMA }}=0$ ARFIMA model reduces to the standard ARMA.

- if $-0.5<d_{\text {ARFIMA }}<0$ the model is anti-persistent, the autocorrelations decay hyperbolically and tend to zero and spectral density is dominated by high frequency components. Shocks can be determined according to the parameter $d_{\text {FIGARCH }}$ profile and the speed of propagation of volatility. If one refers to the analysis of the impulse response functions derived from Eq. (4), we can evaluate

\footnotetext{
${ }^{2}$ A general factor-based solution to the problem of ensuring positive definite variance-covariance matrices was provided by Baba et al. (1989).
} 
$\gamma(1)=\lim _{k \rightarrow+\infty} \sum_{i=0}^{k} \gamma_{i}=\lim _{k \rightarrow+\infty} \lambda_{k}=\mathrm{F}(\mathrm{d}-1,1,1 ; 1) \phi^{-1}(1)[1-\beta(1)]$,

where $\gamma_{k}=\frac{\partial E_{t}\left(\varepsilon_{t+k}^{2}\right)}{\partial v_{t}}-\frac{\partial E_{t}\left(\varepsilon_{t+k-1}^{2}\right)}{\partial v_{t}}$ is the coefficient of the impulse function ${ }^{3}, E_{t}$ is the conditional expectation at the time $t$.

- If $0 \leq d_{F I G A R C H} \leq 1$, then $F(d-1,1,1 ; 1)=0$, from where $\gamma(1)=1$, volatility shocks decay hyperbolically unlike GARCH shocks or decrease exponentially. If $d_{\text {FIGARCH }}=1$ then $F(d-1,1,1 ; 1)=1$ therefore $\gamma(1)=\phi^{-1}(1)[1-\beta(1)]$ and shocks are infinitely persistent.

- If $d_{\text {FIGARCH }}>1$ then the conditional variance is explosive and $\gamma(1) \rightarrow+\infty$. Parameter $d_{\text {ARFIMA }}$ is applied directly on the square of the error unlike parameter $d_{\text {FIGARCH }}$ applying $\sigma_{t}^{2}$ on the FIGARCH model. Also a ARFIMA $(a, 0, m)$ process is reduced to an ARMA $(a, m)$ model while a FIGARCH $(\mathrm{p}, 0, \mathrm{q})$ model can be reduced to a GARCH (p, q) model.

The constant of FIGARCH model can be written as:

$$
w=\phi(L)(1-L)^{d} \sigma^{2} .
$$

Therefore

$$
w=\sigma^{2} \phi(1) \sum_{j=1}^{\infty} \pi_{j}(d) L^{j} .
$$

Also the conditions of positivity of the conditional variance are a problem. Therefore, $\beta \neq 0$, then the parameters of the conditional variance should be checked as follows,

$$
\begin{aligned}
& \lambda_{1}=d-\beta-\phi, \\
& \lambda_{2}=(d-\beta)(\beta-\phi)+\frac{d(d+1)}{2} .
\end{aligned}
$$

For the conditional variance becomes positive, it suffices that all the parameters $\lambda_{k}, k=1, \ldots$ are positive. This amounts to imposing:

$$
0 \leq \phi \leq \beta \leq d<1 .
$$

The multivariate FIGARCH process uses the constant correlation parameterization. This choice is motivated by three considerations:

1- It is the most parsimonious of the available specifications;

2- The variance-covariance matrix is positive definite below weak conditions;

3- Stationary is ensured by restrictions only on the diagonal components of the variancecovariance parameters matrices.

The bivariate constant correlation FIGARCH $(1, \mathrm{~d}, 1)$ process can be represented as:

$$
\begin{aligned}
& h_{j j, t}=\frac{w_{j}}{1-\beta_{j j}(1)}+\lambda_{j j}(L) \varepsilon_{j, t}^{2}, \\
& h_{12, t}=\rho\left[h_{11, t}, h_{22, t}\right]^{\frac{1}{2}},
\end{aligned}
$$

\footnotetext{
${ }^{3} \mathrm{~F}(\mathrm{~d}-1,1,1 ; 1)$ is the hyper-geometric function defined in the general cases by $F(a, b, c ; z)=\sum_{n=0}^{+\infty} \frac{\Gamma(a+k) \Gamma(b+k) \Gamma(c)}{\Gamma(a) \Gamma(b) \Gamma(c+k)} \frac{z^{k}}{k \mathrm{I}}$
} 
where $\lambda_{j j}=1-\left\{\left[\phi_{j j}(L)(1-L)^{d_{j}}\right] /\left[1-\beta_{j j}(L)\right]\right\}$ and $\mathrm{j}=1$,2. It follows from the results of Baillie et al. (1996) that positive confidence in the bivariate diagonal FIGARCH $(1, \mathrm{~d}, 1)$ model is ensured if $|\rho|<1$, $\beta_{j j}-d_{j} \leq\left(\frac{1}{3}\right)\left(2-d_{j}\right)$ and $d_{j}\left[\phi_{j j}-1 / 2\left(1-d_{j}\right)\right] \leq \beta_{j j}\left(\phi_{j j}-\beta_{j}+d_{j}\right)$. The conditional variance defined by this process is stationary for all $0 \leq d_{j} \leq 1$. In what follows, we use a general representation of Eq. (8), which may be represented in the ARFIMA form as:

$$
\Phi(L)\left(\begin{array}{ll}
(1-L)^{d} & 0 \\
0 & (1-L)^{d}
\end{array}\right) \varepsilon_{t}^{2}=w+(I-B(L)) v_{t},
$$

where $\Phi(0)=I$. It is straightforward to test the hypothesis $d_{1}=d_{2}$ within this framework. The parameter $\lambda_{k}$ is based on a relationship between the autoregressive parameters as follows,

$$
\lambda_{k}=\beta \lambda_{k-1}+\left[(k-1-d) k^{-1}-\phi\right] \pi_{k}(d) ; k=2,3 \ldots
$$

where $\pi_{k}(d)=\pi_{k-1}(d)(k-1-d) k^{-1}, k=2,3, \ldots$ are the coefficients of a Maclaurin series expression $(1-L)$. It can be deduced the following inequalities sufficient conditions for the positivity of the conditional variance:

$$
\begin{aligned}
& \beta-d \leq \phi \leq \frac{2-d}{3}, \\
& d\left(\phi-\frac{1-d}{2}\right) \leq \beta \leq \beta(d-\beta+\phi) .
\end{aligned}
$$

The essential condition for positive confidence of the variance-covariance matrix in the FIGARCH process is:

$$
\begin{aligned}
& |\rho|<1, \beta_{j j}-d_{j} \leq(1 / 3)\left(2-d_{j}\right), d_{j}\left[\phi_{j j}-(1 / 2)\left(1-d_{j}\right)\right] \leq \beta_{j j}\left(\phi_{j j}-\beta_{j j}+d_{j}\right), \\
& \phi_{i j}>\beta_{i j}(i, j=1,2 ; i \neq j) .
\end{aligned}
$$

\subsection{Model Specification and Estimation}

We put emphasis on the FIGARCH $(1, d, 0)$ process to investigate the long memory property of volatility of stock index returns. Previous analyses using the GARCH $(p, q)$ model show that most economic time series are well modelled by the low order of $(1,1)$. The model specification is the following:

$$
\begin{aligned}
& y_{t}=\mu+\varepsilon_{t}, \\
& \varepsilon_{t}=\sigma_{t} z_{t}, z_{t} \sim N(0,1), \varepsilon_{\mathrm{t}} / \psi_{t-1} \sim N\left(0, \sigma_{t}^{2}\right), \\
& \phi(L)(1-L)^{d} \varepsilon_{t}^{2}=w+\left[1-\beta_{1}(L)\right] v_{t} .
\end{aligned}
$$

In the previous three equations: $y_{t}$ is the stock return at time $t$, defined by $\ln \left(P_{t} / P_{t-1}\right)$ and $P_{t}$ is the stock price at $t ; \psi_{t-1}$ is the information set at time $t-1 ; \phi(L)$ and $\beta(L)$ are lag polynomials of order 0 and 1, respectively. Eq. (17) can then be rewritten as follows:

$$
\sigma_{t}^{2}=w+\beta_{1} \sigma_{t-1}^{2}+\left[1-\beta_{1}-(1-L)^{d}\right] \varepsilon_{t}^{2} \text {. }
$$


Parameters of Eq. (26) are estimated by the maximum likelihood estimation. The corresponding log likelihood function is maximized as follow:

$$
\log L\left(\theta ; y_{1}, \Lambda, y_{T}\right)=-\frac{1}{2} T \log (2 \pi)-\frac{1}{2} \sum_{t=1}^{T}\left[\log \left(\sigma_{t}^{2}\right)-\frac{\varepsilon_{t}^{2}}{\sigma_{t}^{2}}\right] \text {. }
$$

\subsection{Multivariate GARCH -DCC model}

In this section, we present the two-stage model of the dynamic conditional correlations proposed by Engle (2002). For example, let's consider a vector consisting of any two variables $Y_{t}=\left[y_{1 t} y_{2 t}\right]^{\prime}$. Each variable is a constant function and its own past values. Thus, the reduced form of the autoregressive process is written as:

$$
A(L) Y_{t}=c+\varepsilon_{t} \text { avec } \varepsilon_{t} \rightarrow N\left(0, H_{t}\right), \forall t=1,2, \ldots, T,
$$

where $A(L)$ is the polynomial delay and $\varepsilon_{t}=\left[\varepsilon_{1 t} \varepsilon_{2 t}\right]^{\prime}$ is a vector of residuals from the estimation auto regression process for each variable whose variance-covariance matrix is $H_{t}=\left\{h_{i}\right\}_{t}$ with $i=1,2$.

The DCC-GARCH model can be easily apprehended by rewriting the matrix of variance-covariance $H_{t}$ such as: $H_{t}=D_{t} R_{t} D_{t}$ where $D_{t}=\operatorname{diag}\left\{\sqrt{h_{i t}}\right\}$ is a diagonal matrix of the standard deviations temporally different variable from the estimation of the two previous equations in a process univariate GARCH; $R_{t}=\left\{\rho_{i j, t}\right\}$ which represents the matrix of the conditional correlation parameters. The elements contained in $D_{t}$ are generated in a GARCH $(\mathrm{P}, \mathrm{Q})$ model, which can be formulated as:

$$
h_{i t}=w_{i}+\sum_{p=1}^{P} \alpha_{i p} \varepsilon_{i t-p}^{2}+\sum_{q=1}^{Q} \beta_{i q} h_{i t-q} .
$$

In addition, Engle (2002) adopts a GARCH-type structure in its modelling of the dynamics of correlations. Thus, a DCC process of the order $(\mathrm{M}, \mathrm{N})$ can be described by:

$$
\begin{aligned}
& R_{t}=\left(Q_{t}^{*}\right)^{-1} Q_{t}\left(Q_{t}^{*}\right)^{-1}, \\
& Q_{t}=\left(1-\sum_{m=1}^{M} a_{m}-\sum_{n=1}^{N} b_{n}\right) \bar{Q}+\sum_{m=1}^{M} a_{m}\left(\xi_{t-m} \xi_{t-m}^{\prime}\right)+\sum_{n=1}^{N} b_{n} Q_{t-n},
\end{aligned}
$$

where $\xi_{t}=\left\{\varepsilon_{i t} / \sqrt{h_{i t}}\right\}$ is the vector containing the standardized residuals from the univariate GARCH model estimation, which is the matrix of the conditional variance-covariance of these standardized residuals, whereas $Q_{t}=\left\{q_{i j, t}\right\}$ is the matrix of the unconditional variance-covariance, which are temporally invariant. The parameters $\left(a_{m} ; b_{n}\right)$ are supposed to intercept, respectively, the effects of the shock and delay the dynamic correlations on the level of recent contemporary. $Q_{t}^{*}$ is a diagonal matrix containing the square root of the main diagonal elements of $Q_{t}$. According to our example this matrix is written as:

$$
Q_{t}^{*}=\left(\begin{array}{ll}
\sqrt{q_{11}} & \\
0 & \sqrt{q_{22}}
\end{array}\right)
$$

where $\rho_{12, t}=\frac{q_{12, t}}{\sqrt{q_{11, t} q_{22, t}}}$ is the dynamic conditional correlations which are the matrix elements $R_{t}$ whose main diagonal consists of 1 . The model parameters are estimated by the DCC method of maximum likelihood. Engle (2002) showed that the log-likelihood function can be expressed as: 


$$
L=-\frac{1}{2} \sum_{t=1}^{T}\left\{2 \log (2 \pi)+2 \log \left|D_{t}\right|+\log \left|R_{t}\right|+\xi_{t} R_{t}^{-1} \xi_{t}\right\} .
$$

\section{Data and Results}

\subsection{Data and descriptive statistics}

The data comprise daily total return indices calculated by "yahoo.finance" for markets of developed countries. We have chosen WTI for the American markets. As far as the European countries are concerned, Brent oil price. The sample starts in January 1996 and ends in March 2014, yielding 2137observations for each series.
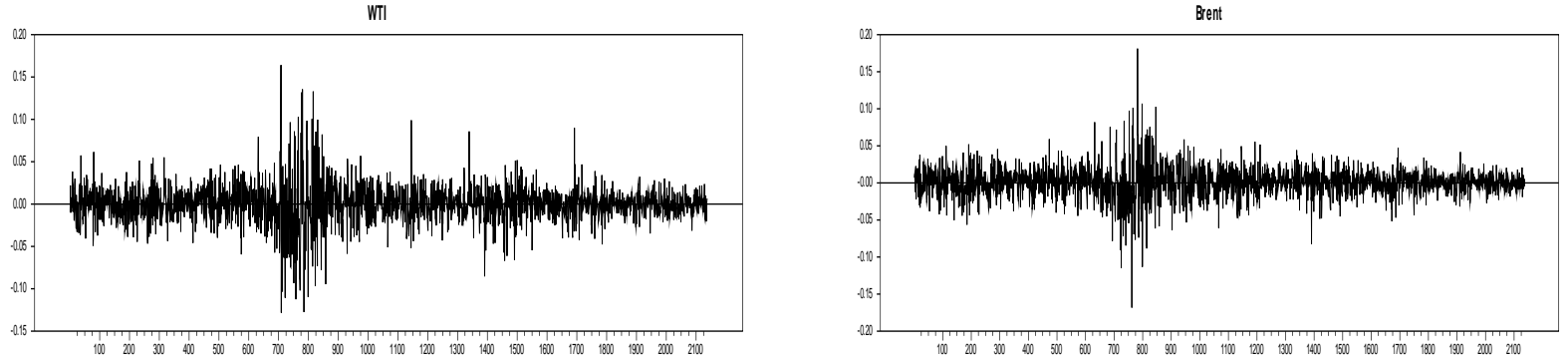

Fig. 1. The oil price returns

Fig. 1 indicates the trend of each oil price returns, for example large changes tend to be followed by large changes in numbers and also sign and small changes tend to be followed by small changes for all cases. There is some evidence of volatility clustering; that is, periods of high volatility followed by periods of tranquility. There is also confirmation of chocks in volatility.

\section{Table 1}

Descriptive Statistics

\begin{tabular}{lcc}
\hline & WTI & Brent \\
\hline Obs & 2137 & 2137 \\
Mean & 0.00021 & 0.00026 \\
St. dev. & 0.023 & 0.0209 \\
Skewness & 0.102 & 0.0039 \\
Kurtosis & 6.059 & 7.421 \\
JB & 3273.58 & 4904.14 \\
\hline
\end{tabular}

Note: this table presents the descriptive statistics. $\left(^{* * *}\right)$ indicate the rejection of the null hypothesis of associated statistic tests at the $1 \%$ level.

Table 1 reports descriptive statistics of the WTI and Brent oil prices returns series. Mean returns were very small relative to the standard deviations. All the values of the Skewness statistic were positive as well as more pronounced in the WTI oil price return, suggesting a greater probability of large decreases in index returns compared with both series of oil price. The Skewness values demonstrate that the marginal distributions are asymmetrical to the left. The high values of the Kurtosis for all the returns' series are not normally distributed (Skewness $\neq 0$ and Kurtosis $>3$ ). We also notice as well high kurtosis values, generally superior to 3 . These suggest that the distributions of the different markets' returns are leptokurtic. It is fairly empirical to show that $w>0,0<d<1-2 \phi_{1}$ and $0 \leq \beta_{1} \leq \phi_{1}+d$ are sufficient to certify that the conditional variance of the FIGARCH $(1, \mathrm{~d}, 1)$ model represents the persistency of volatility in the stock return only for the studied period. This indicates the rejection of hypothesis efficiency of the oil price markets in this period indicating the effect of recent financial crisis. For the FIGARCH process, the estimate of long memory component, $d$, is found to be significantly different from zero and is within the theoretical value, representing that the volatility exhibits a long memory model in the oil prices markets. This result shows the importance of modelling a long memory in volatility and proposes that future volatility depends on its past realizations and therefore, is predictable. This consequence also supports the findings of other studies on two emerging and developed markets. 
We report the estimation results in Table 2. When looking at the variance equation coefficients, we see that the fractional differencing parameters (d) are highly significant in the two cases studied.

Table 2

Estimation results of FIGARCH models

\begin{tabular}{lcc}
\hline & WTI & Brent \\
\hline$\mu$ & $7.53 .10^{-4}(0.03)$ & $5.91 .10^{-4}(0.09)$ \\
$w$ & $-0.016(0.49)$ & $0.014(0.53)$ \\
$\mu$ & $-6.717 .10^{-7}(0.86)$ & $-7.36 .10^{-6}(0.38)$ \\
$w$ & $2.51 .10^{-5}(0.02)$ & $1.90 .10^{-5}(0.07)$ \\
$\beta_{1}$ & $0.761(0.00)$ & $0.626(0.00)$ \\
$\Phi_{1}$ & $0.437(0.00)$ & $0.246(0.01)$ \\
$d$ & $0.536(0.00)$ & $0.413(0.00)$ \\
\hline Note: the p-values are reported in the parentheses below corresponding components estimates
\end{tabular}

The persistency is the strongest in the WTI oil price, than the Brent. This effect is consistent with the values of $d$ estimated in FIGARCH. The long-range dependence components are 0.536 for the WTI oil market and 0.413 for the Brent. During the recent period, the market demonstrates the greatest strength of a long-range dependence. The weak performance of oil price market has made investors flee the oil price market. As a result, most of the markets members tend to pursue the trends and quit leave depart the oil price market. This is dependable in the study of Shiller (2000) who argued that generally market participants are not 'smart investors' but fairly followers of trends and fashions. This may be caused by the heterogeneous participants' reactions in respect to their optimistic or pessimistic visions of the future view of oil price markets. The estimated models are steady with the trend of our general Hurst estimations in all the periods. To determine the transmission of shocks between oil price markets we will use the DCC-MGARCH approach.

To measure the persistence of shocks on the volatility of the bank returns, we used the GARCH $(1,1)^{4}$. Table 3 shows that coefficients $\alpha_{11}$ and $\beta_{11}$ for WTI $(0.048+0.66)<1$ and $\alpha_{22}$ and $\beta_{22}$ for Brent $(0.035+0.628)<1$ are stationary and positive for the WTI and Brent oil price markets. According to the estimate of the GARCH model, we note a high persistence of shocks for WTI oil price $(\alpha+\beta$ tends to 0.708 ) is more persistent than the Brent ( $\alpha+\beta$ tends to 0.663 ). Table 3 shows that the correlation coefficients $\left(\bar{\rho}_{i j}\right)$ are pretty small, and all are below 0.5 , indicating that the selected conditioning variables contain sufficiently orthogonal information.

Table 3

Results of the GARCH-DCC $(1,1)$ Bivariate $^{5}$ models

\begin{tabular}{lcc}
\hline & WTI-Brent & p-value \\
\hline C1 & 0.00017 & 0.85 \\
C2 & 0.000034 & 0.96 \\
$w_{1}$ & 0.00026 & 0.042 \\
$w_{2}$ & 0.00027 & 0.072 \\
$\alpha_{11}$ & 0.048 & 0.00 \\
$\alpha_{22}$ & 0.035 & 0.16 \\
$\beta_{11}$ & 0.66 & 0.00 \\
$\beta_{22}$ & 0.628 & 0.00 \\
$\alpha_{c}$ & 0.188 & 0.00 \\
$\beta_{c}$ & 0.224 & 0.00 \\
\hline
\end{tabular}

\footnotetext{
${ }^{4}$ The GARCH(1,1) process can be written as follows : $h_{t}=\alpha_{0}+\alpha_{1} \varepsilon_{t-1}^{2}+\beta_{1} h_{t-1}$

${ }^{5}$ The choice of this framework can be justified by the results of Bivariate GARCH models. These results show that there exists a phenomenon of contagion between markets studied coming directly from WTI oil price market to the Brent.
} 
We find $b_{n}$ being greater than $a_{m}$, under restriction that coefficients and $\alpha_{c}+\beta_{c}<1$. The evidence from these results suggests that a big shock just causes a small correction in the approaching mutual fluctuation (or covariance) between the markets.
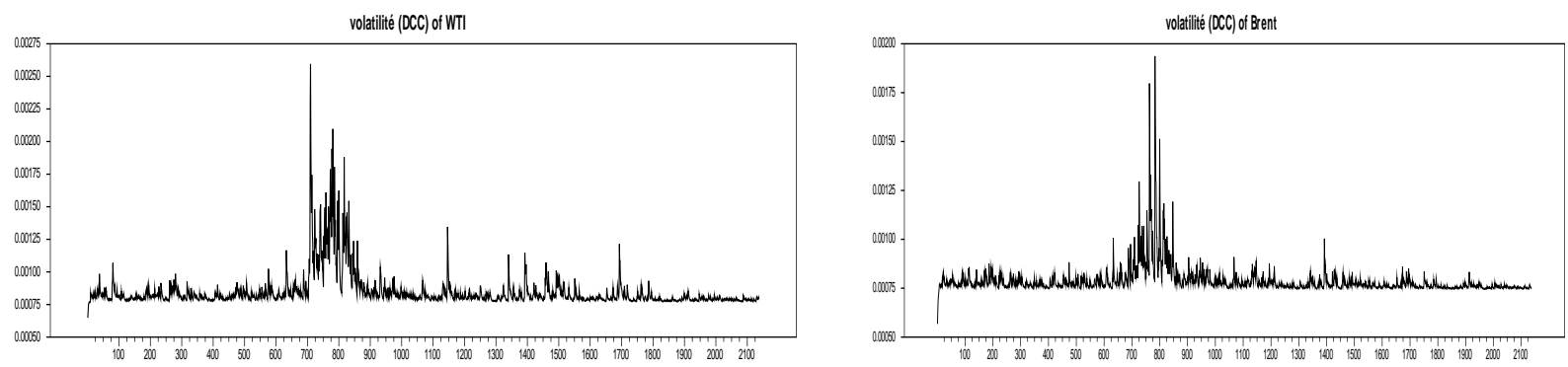

Fig. 2. Oil price volatility

The graphs Fig. 2, from the GARCH $(1,1)$ show a high market volatility at specific dates: September 2008, March 2009, April 2010. In addition, most of these dates coincide with the oil prices markets in two indices where returns on indices are negative. Everything confirms the asymmetric behavior of the volatility shocks. Furthermore, this observation of high volatility suggests a change in the trend of the variance in these particular periods and the existence of break points (ICSS algorithm). Sansó et al. (2004) could detect multiple break points for each series. The series having generally distinct break dates do not prevent the existence of joint periods corresponding to the major events showing some chocks (Fig. 2).

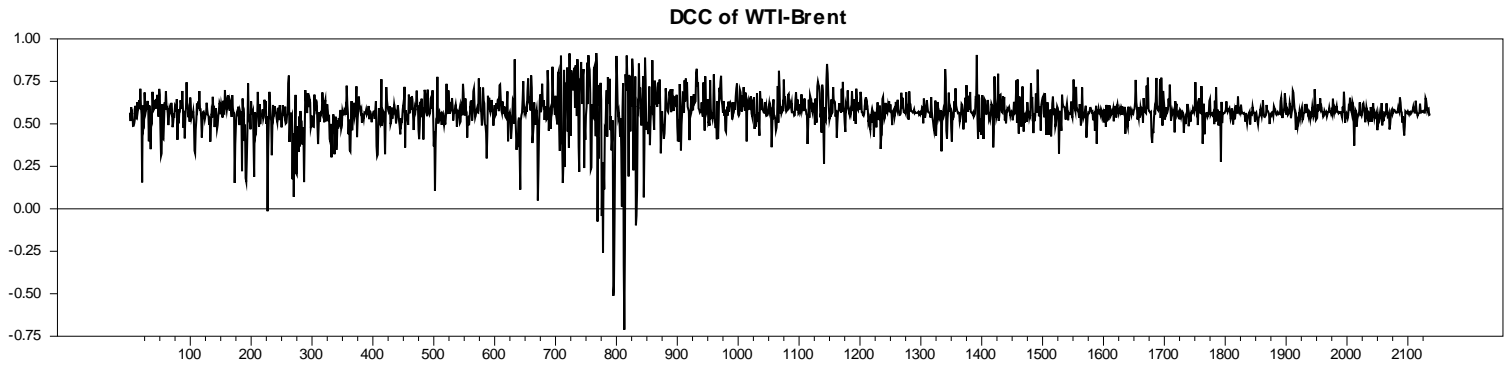

Fig. 3. Dynamic conditional correlations

By examining Fig. 3, we can say that the evolution of correlations between the WTI and Brent oil prices markets returns with the other bank developed markets leads to the following observations:

The conditional correlations between the WTI and Brent oil price markets are sometimes positive. However, it is almost clear that by the end of the financial crisis, the correlations considerably increased to exceed $80 \%$ for USA and European markets. The conditional correlation has been much more pronounced since the beginning of the crisis in 2007. The coefficients are dynamic and reached a peak in 2009. We conclude that there is a contagious effect of the WTI oil price on the developed oil price markets (European markets). According to Kaminsky and Reinhart (1999, 2000) and Broner and Gelos (2003), it is possible to see that this contagion is triggered by the oil price channel which reflects the relations between the developed countries in terms of equities or loan portfolios.

\section{Conclusions}

Our objectives were to develop models for agile processes in closely related markets volatility, while recognizing the long memory fraction. The volatility is still little consensus on the appropriate methodology for testing fractional cointegration, especially in this case when it relates to latent processes. The contribution of this paper was to demonstrate the feasibility of estimating and testing 
models cointegrated bivariate FIGARCH, at least in the case in which usual attention was limited to a unit cointegrating vector.

This article examines the rapid change of volatility in the WTI and Brent oil price using the multipleshift FIGARCH model. Our empirical results designated numerous structural changes in volatility particularly during the economic and currency crisis. We found that the standard FIGARCH models had an excess of estimation evidencing the long-persistence clustering volatilities in the oil price market. Though, our empirical results evidenced a substantial decrease in volatility persistence after the insertion of structural changes through the Asian economic and currency crises. Finally, the fractionally integrated models with structural changes executed better in the estimated and specified evaluations. The price of a barrel of crude is a key indicator, we saw all the differences between Brent oil and WTI, insofar know that there are many varieties called conventional oil (more than 150 types of oil). In fact some oils are richer in heavy products and sulfur container varies, the more it is high unless it is of good quality, which impacts the costs associated with refining.

\section{References}

Askari, H., \& Khrichene, N. (2008). Oil price dynamics (2002-2006). Energy Economics, 30, 21342153.

Baba, Y., Engle, R.F. Kraft, D.F. \& Kroner, K.F. (1989). Multivariate simultaneous generalized ARCH. Department of Economics, University of California, San Diego., Discussion paper 89-57.

Baillie, R. T., Bollerslev, T., \& Mikkelsen, H. O. (1996). Fractionally integrated generalized autoregressive conditional heteroskedasticity. Journal of Econometrics, 74(1), 3-30.

Bollerslev, T. (1986). Generalized autoregressive conditional heteroskedasticity. Journal of Econometrics, 31(3), 307-327.

Breidt, F. J., Crato, N., \& De Lima, P. (1998). The detection and estimation of long memory in stochastic volatility. Journal of Econometrics, 83(1), 325-348.

Broner, F., \& Gelos, G. (2003). Testing the portfolio channel of contagion: the role of risk aversion. Universitat Pompeu-Fabr.

Engle, R. (2002). Dynamic conditional correlation: A simple class of multivariate generalized autoregressive conditional heteroskedasticity models.Journal of Business \& Economic Statistics, 20(3), 339-350.

Engle, R. F. (1982). Autoregressive conditional heteroscedasticity with estimates of the variance of United Kingdom inflation. Econometrica: Journal of the Econometric Society, 50(4), 987-1007.

Hosking, J. (1981). Fractional Differencing. Biometrika, 68(1), 165-167.

Huang, Y.C., Li, C., \& Ma, W.F. (2005). The dilemma and outlet of China's oil price risk management. World Economy Study, 10, 22-26.

Granger, C.W.J, \& Joyeux, R. (1980). An introduction to long memory time series models and fractional differencing. Journal of Time Series Analysis, 1(1), 15-29.

Kaminsky, G. L., \& Reinhart, C. M. (1999). The twin crises: the causes of banking and balance-ofpayments problems. American Economic Review, 89(3), 473-500.

Kaminsky, G. L., \& Reinhart, C. M. (2000). On crises, contagion, and confusion. Journal of International Economics, 51(1), 145-168.

Larsson, K. \& Nossman, M. (2011). Jumps and stochastic volatility in oil prices: Time series evidence. Energy Economics, 33, 504-514.

Li, P.M., Jia, M., \& Zhang, G.Y. (2005). China's strategies for the high oil price. Macroeconomics. 12, 8-14 (in Chinese).

Sansó, A., Aragó, V., \& Carrion, J. L. (2004). Testing for changes in the unconditional variance of financial time series. Revista de Economía financiera,4, 32-53.

Shiller, R. J. (2000). Measuring bubble expectations and investor confidence.The Journal of Psychology and Financial Markets, 1(1), 49-60. 